\title{
Genome-Wide Association Studies and Aggressive Surgery Toward Individualized Prevention, and Improved Local Control and Overall Survival for Gastric Cancer
}

\author{
Dimitrios H. Roukos, MD \\ Surgical Oncology Research Unit, Biobank Personalized Cancer Biomedicine, Department of Surgery, Ioannina University \\ School of Medicine, Ioannina 45110, Greece
}

Prevention and improved treatment can dramatically improve the poor prognosis of gastric cancer. Two pivotal studies in biomedical sciences and clinical research for the identification of genetic-factors-based primary prevention and surgical treatment of stomach cancer have most recently been reported from Japan. ${ }^{1,2}$ What are the clinical implications of these studies?

In the field of risk-stratification-based personalized prevention, a novel gastric cancer candidate gene has been identified. A new genome-wide association study (GWAS) published in the June issue of Nature Genetics has demonstrated that genetic variants in the common, lowpenetrance PSCA gene are associated with increased risk of sporadic diffuse-type gastric cancer. ${ }^{1}$ Could genetic testing for these risk variants, or other new variants which will be identified by GWAS in the future, be used in the clinic?

In the field of treatment, the results of a phase III randomized trial comparing D2 gastrectomy alone or D2 plus para-aortic lymph nodal dissection (PAND) in patients with advanced resectable gastric cancer have been reported in the July 31 issue of NEJM. ${ }^{2}$ The overall 5-year survival rate was around $70 \%$, similar in both groups. This excellent result has been achieved by surgery alone. Given the poor prognosis of the disease in the West, despite the addition of modern adjuvant treatment to surgery, what are the applications of these Japanese results in the USA and Europe ${ }^{3,4}$

Worldwide, gastric cancer, despite its declining incidence, still remains an important health problem, being the fourth most common cancer and the second most common

(C) Society of Surgical Oncology 2009

Published Online: 24 January 2009

D. H. Roukos, MD

e-mail: droukos@cc.uoi.gr cause of death. There is wide geographical variation. The annual age-standardized incidence rate in Japan ranges from 50 to 80 per 100,000 among men and from 20 to 30 per 100,000 among women while in the USA the figures are about one-tenth of those: 5.9 per 100,000 for men and 2.5 per 100,000 for women. ${ }^{5}$ We need effective prevention, early detection, and treatment strategies to reduce morbidity and mortality of the disease. ${ }^{6}$

\section{RISK-STRATIFICATION-BASED PREVENTION}

Despite efforts, primary prevention for clinical practice has not yet been developed. A key in designing prevention strategies is the discrimination of general population and individual persons into high-, intermediate-, and low-risk categories for developing gastric cancer. More aggressive preventive interventions, such as prophylactic surgery and drugs, may be justifiable for high-risk people, whereas lifestyle recommendations are sufficient for low-risk subjects. What are the perspectives toward risk assessment and targeted prevention?

\section{Familial and Sporadic Gastric Cancer}

On the basis of family history and heritability, gastric cancer can be discriminated into familial cancer and sporadic cancer (absence of family history). In 1998 a breakthrough came: inherited mutations in the high-penetrance $\mathrm{CDH} 1$ gene cause hereditary diffuse gastric cancer (HDGC). ${ }^{7}$ Following this discovery and the current available genetic testing result, familial gastric cancer is classified into $C D H 1$-positive and $C D H 1$-negative cancer. Individuals with inherited $\mathrm{CDH} 1$ mutations face a very high lifetime risk of around $75 \%$ for gastric cancer, and 
additionally for women a $40 \%$ risk for lobular breast cancer. $^{8}$ The E-cadherin protein encoded by the $C D H 1$ gene is important in the molecular connections between adjacent cells in the stomach, the breast, and also other areas of the body. Loss of E-cadherin function-associated with $\mathrm{CDH}$ mutations is seen in diffuse-type gastric cancer and also invasive lobular breast cancer. ${ }^{9}$

In about $70-90 \%$ of patients gastric cancer is sporadic whereas in $10-30 \%$ it is familial. ${ }^{10}$ Among familial gastric cancer cases, only about $1-3 \%$ are carriers of mutations in CDH1. ${ }^{11}$

Targeted effective prevention in clinical practice is currently feasible only for the high-risk subpopulation with inherited $C D H 1$ mutations. ${ }^{12}$ Within a decade after identification of $C D H 1$, prophylactic total gastrectomy has been increasingly used in clinical practice. It is thought to be a safe and highly effective preventive intervention with acceptable level of quality of life for $\mathrm{CDH} 1$ mutation carriers. $^{13-15}$

\section{Prevention of Sporadic Gastric Cancer}

It is thought that interaction between Helicobacter pylori infection and dietary risk factors leads to gastric carcinogenesis. Therefore, prevention randomized trials in high-risk populations, including Japan, considering these factors, would be effective. ${ }^{5}$ However, such prevention trials with $H$. pylori eradication and nutritional interventions in high-risk populations were negative. ${ }^{16}$ How can this failure be explained?

Two key issues in the design of these trials have not been considered: Lauren classification ${ }^{17}$ and genetic factors. About four decades after the introduction of Lauren classification, a latest GWAS confirms that the intestinal and diffuse types are two distinctly different subtypes of gastric cancer. ${ }^{1}$ The intestinal type seems to be associated with $H$. pylori infection, which may lead to atrophic gastritis, intestinal metaplasia, dysplasia, and invasive adenocarcinoma, and is particularly common in some highrisk geographic regions, including Japan. In contrast, the diffuse type develops through different pathway, and the genetic background is more important than for the intestinal type, seems unrelated to the presence of $H$. pylori, and has a much more uniform geographical distribution. ${ }^{6}$ Genetic factors have an important role in tumorigenesis. However, until recently no susceptibility loci with large effects-beyond $\mathrm{CDH} 1$-on gastric carcinogenesis has been identified and validated.

\section{Whole-Genome Scan}

Genetic variation-differences in both the coding and noncoding portions of our DNA - is what makes each of us unique. It also can contribute to our personalized susceptibility to disease. Exhaustive analysis of human single-nucleotide polymorphisms (SNPs) has led to the identification of interesting SNP markers for certain disorders. Genetic variants include, beyond SNPs, also copy number variations (CNVs) - gain or loss of segments of genomic DNA relative to a reference-which have also been shown to be associated with several complex and common disorders such as cancer. ${ }^{18}$ There has been an explosion in biomedical research for the identification of genetic-variation-based personalized management of complex diseases. Over the last 2 years GWAS have identified more than 100 new chromosomal regions at which more than 165 novel DNA variants influence risk of common human diseases and clinical phenotypes. $^{19}$

Recently the Study Group of Millennium Genome Project for Cancer reported the results of a new GWAS with the identification of the PSCA gastric cancer susceptibility gene. ${ }^{1}$ This two-stage GWAS used 85,576 SNPs on 188 patients with diffuse gastric cancer and 752 references in stage 1 and 2,753 SNPs on 749 patients and 750 controls in stage 2 in Japan, and identified an association of a SNP (rs2976392) in the PSCA with diffuse gastric cancer. Resequencing of the PSCA region in the affected individuals revealed a number of SNPs that increase a person's risk for diffuse gastric cancer even if there is no family history (sporadic cancer). In a replication study in Korean populations the authors also found an association of PSCA SNPs with diffuse gastric cancer.

Unsurprisingly, the relative risk of all the genetic variants in PSCA in this study was smaller than $2 .{ }^{1}$ Indeed, this finding is consistent with all GWAS performed for cancer or other common diseases. ${ }^{19}$ Although the flood of discoveries through whole-genome analysis is impressive, there is no clinical implication because of small effects of these risk variants. ${ }^{20}$ Thus, it is confirmed how hard the translation of basic-science breakthroughs into clinical applications is, revealing a widening gap between basic and clinical research and medical practice. ${ }^{21}$

\section{SURGERY, LOCAL CONTROL, AND SURVIVAL}

Locoregional tumor control is important for the clinical outcomes of patients with solid tumors, including gastric cancer and breast cancer. Preventing local and nodal failures, when they occur as first and isolated events, due to appropriate surgery, may improve overall survival. ${ }^{4,22}$

Sasako et al. performed a surgical study in 24 specialized hospitals in Japan. ${ }^{2}$ The 523 enrolled patients all had an advanced resectable (T2, T3 or T4) gastric tumor and underwent D2 surgery alone or D2 plus PAND. Surgery- 
related complications rates were higher in the D2 plus PAND group (28.1\%) than in the D2 group (20.9\%). Mortality rate within 30 days after surgery was $0.8 \%$ in each group. There was no significant difference in recurrencefree survival or overall survival between the two groups.

Given that no patient received adjuvant treatment, survival rate in this study reflects the therapeutic ability of surgery alone to control the disease. The 5-year survival rate of about $70 \%$ in each group is the best ever reported by a well-designed and well-conducted randomized trial. This study performed by the Japan Clinical Oncology Group provides several robust conclusions: First, the very extensive D2 plus PAND increases surgical morbidity, even when it is performed by experienced surgeons, without any survival benefit. Thus, this surgical procedure definitively remains out of clinical practice, particularly in the West. Second, advanced resectable gastric cancer is a local disease for most patients. Thus, appropriate local control by standardized Japanese-type D2 surgery for Japanese population provides long-term survival. Following recent evidence that adjuvant chemotherapy after D2 surgery significantly improves survival, D2 plus S-1 chemotherapy has been the standard of care in Japan. ${ }^{23}$

What are the implications of the study by Sasako et al. in the Western population? Pharmaceutical industry supports research on highly expensive adjuvant empirical and targeted therapies rather than local control with D2 surgery. As a result, adjuvant postoperative chemoradiotherapy in the USA or perioperative chemotherapy in Europe with limited D1 lymphadenectomy are common on the basis of positive results of INT-0116 trial and MAGIC trial, respectively. ${ }^{3,24}$ However, despite high-cost adjuvant treatment, survival in these trials is approximately half of that reported by Sasako et al. with D2 surgery alone.

How can the huge difference in survival rates between Japanese and Western populations be explained? Differences in genetic background between the populations and the absence of standardized D2 lymphadenectomy in the Western randomized trials may explain this survival difference.

\section{FUTURE PERSPECTIVES}

If research on personalized prevention and treatment of gastric cancer could be translated into practice, it would dramatically improve clinical outcomes. GWAS hold promise to identify genetic risk variants which could be used as prognostic and predictive genetic tests to tailor an optimal preventive or therapeutic intervention in individual persons or patients. However, the achievement of this goal is extremely difficult and many hurdles remain to be overcome, as revealed now by the latest data on personal genomics, genetics, and GWAS. ${ }^{19,25,26}$
As mentioned above, the SNPs in the PSCA gene identified by the Japanese GWAS only modestly increase the risk of diffuse gastric cancer and thus a genetic testing with these variants has no clinical application. ${ }^{1,20}$ Future GWAS using modern genotyping platforms with more than 1 million SNPs or CNVs will be able to identify a dozen or more further genetic risk variants. However, because of small effect of these variants in gastric cancer risk, functional studies will be required to explore possible multiplicative interactions of these DNA variants. Ultimately, large, prospective, population-based studies recording family and medical history and genetic and environmental factors, including H. pylori infection and dietary habits, will lead to gastric cancer risk assessment for both general population and individual persons. This stratification and potential response prediction to various preventive interventions may lead to true personalized gastric cancer prevention. ${ }^{26}$

Of much more clinical interest would be the potential discovery by new GWAS of novel gastric cancer susceptibility genes with large effects, such as a high-penetrance gene for intestinal-type cancer such as the $\mathrm{CDHl}$ gene for hereditary diffuse gastric cancer. Indeed, in this case, carriers of mutations in a novel high-penetrance gene would face a very high risk of gastric cancer, and aggressive prevention would be justified.

Currently, standard treatment of advanced resectable gastric cancer includes complete surgical resection (R0) of the tumor and adjuvant chemotherapy or chemoradiotherapy. ${ }^{3,23,24}$ To test whether the excellent Japanese survival results can be achieved in the West we need a new largescale randomized trial with an appropriate local control due to a Japanese-style standardized D2 surgery followed by modern adjuvant treatment.

The major goal for personalized treatment could be achieved by the discovery of predictive markers to tailor the therapeutic approach with the highest response probability and lowest possible adverse effects. Biomedical research on molecular profiling, personal genomics, and pharmacogenomics provide fascinating promises towards personalized local and distant control of solid tumors, including gastric cancer and breast cancer, but there are challenges and hurdles to overcome. ${ }^{25,27,28}$

\section{CONCLUSIONS}

Effective prevention of gastric cancer is currently feasible for high-risk $\mathrm{CDH} 1$ mutation carriers. However, they account for only $1-3 \%$. For the vast majority of the remaining patients, GWAS hold major promises, particularly if they lead to the discovery of further highpenetrance genes. Although identification of further low- 
penetrance loci, beyond PSCA, is expected from new GWAS, there will be limitations for their clinical use because of small effects of genetic variants (SNPs/CNVs) in gastric cancer risk.

Prevention of locoregional recurrence, when they occur as first, isolated event, due to appropriate standardized D2 surgery for advanced resectable gastric cancer, may improve both adjuvant treatment efficacy and overall survival. Western randomized trials with Japanese-style D2 surgery plus adjuvant empirical cytotoxic and targeted treatment is warranted.

\section{REFERENCES}

1. Study Group of Millennium Genome Project for Cancer, Sakamoto H, Yoshimura K, Saeki N, Katai H, Shimoda T, Matsuno $\mathrm{Y}$ et al. Genetic variation in PSCA is associated with susceptibility to diffuse-type gastric cancer. Nat Genet. 2008;40(6):730 40.

2. Sasako M, Sano T, Yamamoto S, Kurokawa Y, Nashimoto A, Kurita A, et al. for Japan Clinical Oncology Group. D2 lymphadenectomy alone or with para-aortic nodal dissection for gastric cancer. N Engl J Med. 2008;359(5):453-62.

3. Cunningham D, Allum WH, Stenning SP, Thompson JN, Van de Velde CJ, Nicolson M, et al. Perioperative chemotherapy versus surgery alone for resectable gastroesophageal cancer. $N$ Engl $J$ Med. 2006;355:11-20.

4. Liakakos T, Roukos DH. More controversy than ever-challenges and promises towards personalized treatment of gastric cancer. Ann Surg Oncol. 2008;15(4):956-60.

5. Forman D, Pisani P. Gastric cancer in Japan-honing treatment, seeking causes. $N$ Engl J Med. 2008;359(5):448-51.

6. Roukos DH. Current status and future perspectives in gastric cancer management. Cancer Treat Rev. 2000;26(4):243-55.

7. Guilford P, Hopkins J, Harraway J, McLeod M, McLeod N, Harawira $\mathrm{P}$, et al. E-cadherin germline mutations in familial gastric cancer. Nature. 1998;392(6674):402-5.

8. Pharoah PD, Guilford P, Caldas C, International Gastric Cancer Linkage Consortium. Incidence of gastric cancer and breast cancer in $\mathrm{CDH} 1$ (E-cadherin) mutation carriers from hereditary diffuse gastric cancer families. Gastroenterology. 2001;121(6): 1348-53.

9. Brooks-Wilson AR, Kaurah P, Suriano G, Leach S, Senz J, Grehan N, et al. Germline E-cadherin mutations in hereditary diffuse gastric cancer: assessment of 42 new families and review of genetic screening criteria. J Med Genet. 2004;41:508-17.

10. Lichtenstein P, Holm NV, Verkasalo PK, Iliadou A, Kaprio J, Koskenvuo $\mathrm{M}$ et al. Environmental and heritable factors in the causation of cancer-analyses of cohorts of twins from Sweden, Denmark, and Finland. N Engl J Med. 2000; 343(2):78-85.
11. Barber M, Fitzgerald RC, Caldas C. Familial gastric canceraetiology and pathogenesis. Best Pract Res Clin Gastroenterol. 2006;20(4):721-34.

12. Roukos DH, Murray S, Briasoulis E. Molecular genetic tools shape a roadmap towards a more accurate prognostic prediction and personalized management of cancer. Cancer Biol Ther 2007;6(3):308-12.

13. Norton JA, Ham CM, Van Dam J, Jeffrey RB, Longacre TA, Huntsman DG, et al. CDH1 truncating mutations in the E-cadherin gene: an indication for total gastrectomy to treat hereditary diffuse gastric cancer. Ann Surg. 2007;245:873-9.

14. Roukos DH, Kappas AM, Tsianos E. Role of surgery in the prophylaxis of hereditary cancer syndromes. Ann Surg Oncol. 2002;9:607-9.

15. Ziogas D, Baltogiannis G, Fatouros M, Roukos DH. Identifying and preventing high-risk gastric cancer individuals with $\mathrm{CDH} 1$ mutations. Ann Surg. 2008;247(4):714-5.

16. Taylor PR. Prevention of gastric cancer: a miss. J Natl Cancer Inst. 2007;99(2):101-3.

17. Lauren $P$. The two histological main types of gastric carcinoma: diffuse and so-called intestinal-type carcinoma. Acta Pathol Microbiol Scand. 1965;64:31-49.

18. Lee C, Morton CC. Structural genomic variation and personalized medicine. $N$ Engl J Med. 2008;358(7):740-1.

19. Manolio TA, Brooks LD, Collins FS. A HapMap harvest of insights into the genetics of common disease. J Clin Invest. 2008;118:1590-605.

20. Hunter DJ, Khoury MJ, Drazen JM. Letting the genome out of the bottle-will we get our wish?. N Engl J Med. 2008;358(2):105-7.

21. Butler D. Translational research: crossing the valley of death. Nature. 2008;453(7197):840-2.

22. Punglia RS, Morrow M, Winer EP, Harris JR. Local therapy and survival in breast cancer. $N$ Engl J Med. 2007;356:2399-40.

23. Sakuramoto S, Sasako M, Yamaguchi T, Kinoshita T, Fujii M, Nashimoto A, et al. Adjuvant chemotherapy for gastric cancer with S-1, an oral fluoropyrimidine. $N$ Engl J Med. 2007;357: 1810-20.

24. Macdonald JS, Smalley SR, Benedetti J, Hundahl SA, Estes NC, Stemmermann GN, et al. Chemoradiotherapy after surgery compared with surgery alone for adenocarcinoma of the stomach or gastroesophageal junction. N Engl J Med. 2001;345:725-30.

25. Roukos DH, Lykoudis E, Liakakos T. Genomics and challenges toward personalized breast cancer local control. J Clin Oncol 2008;26(16):4360-1.

26. Roukos DH. Assessing both genetic variation (SNPs/CNVs) and gene-environment interactions may lead to personalized gastric cancer prevention. Expert Rev Mol Diagn. 2009;9(1):1-6.

27. Roukos DH. Innovative genomic-based model for personalized treatment of gastric cancer: integrating current standards and new technologies. Expert Rev Mol Diagn. 2008;8(1):29-39.

28. Roukos DH. Genetics and genome-wide association studies: surgery-guided algorithm and promise for future breast cancer personalized surgery. Expert Rev Mol Diagn. 2008;8(5):587-97. 